\title{
Misuse of antibiotics: A potential threat
}

\author{
Neha Saini ${ }^{1}$, Varun Saini ${ }^{2}$, Priya Wadhwa Mehta ${ }^{3}$ \\ ${ }^{I}$ (Department of Periodontics Jaipur Dental College, Jaipur,India) \\ ${ }^{2}$ (Department of Prosthodontics, RR Dental College, Udaipur, India) \\ ${ }^{3}$ (Department of Periodontics, VSPM Dental College \& Research Centre,Nagpur,India)
}

\begin{abstract}
:
Introduction: Antibiotics are invaluable adjuncts in the management of orofacial infections. Role of dentists \& injudicious use of antibiotics resulting in antimicrobial resistance is unknown. Therefore a survey was conducted.
\end{abstract}

Aims \& Objectives: To assess the types and frequency of antibiotic prescriptions, by dental practitioners in the state of Rajasthan, India.

Method: A two page questionnaire was mailed to 525 dental practitioners \& the results were assessed.

Results: A total of 500 dentists responded to the questionnaire .

Discussion: Confusion about prescribing antibiotics \& inappropriate prescribing practises, however are reported by dentists (Steed et al 1997). Patients not finishing the prescribed doses, wrong prescription, inefficient tools for diagnosing \& preventing drug resistance, lack of Govt. commitment to address these issues are various factors that may affect development of antibiotic resistant microorganisms. (Loesche 1996)

Conclusion: Amoxicillin - Clavulunic acid combination was the most preferred antibiotic prescribed by dental practitioners for orodental infections followed by amoxicillin \& ofloxacin ornidazole combination. Discrepancies were noted in the prophylactic use of antibiotics, which supports the need for educational initiatives to promote rational use of antibiotics in dentistry.

Keywords: misuse, antibiotics, dental practices, orofacial infections

\section{Introduction}

Various periodontal diseases are caused by bacterial infections. Antibiotics are valuable adjuncts in the management of orofacial infections. The evidence is at present insufficient to recommend systemic antimicrobial therapy as monotherapy ( i.e. as stand alone treatment without scaling \& root planing or surgery) . Antibiotics are not a substitute for definitive treatment but their use can shorten infection periods $\&$ minimise associated risks such as the spread of infection to adjacent anatomical spaces or systemic involvement [1]. The prescription of antibiotics in dentistry is relatively small but nonetheless significant. Injudicious prescription of antibiotics by doctors, healthcare workers \& patients using it on their own has resulted in emerging microbial resistance to various antibiotics \& this trend is likely to continue given the widespread use of antibiotics [2]. With the emergence of bacterial species resistant to antibiotics there is a need to become vigilant about their prescription and with this, an urgent requirement for both professional and public understanding of the appropriate use of this life-saving component of treatment [3].Minimizing the occurrence of antibiotic misuse and abuse has global implications for the containment of resistant bacterial strains [4]. WHO has considered the emergence of resistance microorganisms as a serious global health concern [5].

A survey in the form of a questionnaire was conducted to assess the current antibiotic use by general dental practitioners \& specialists. This paper discusses some of the current understanding of prescribing practices by dentists in the state of Rajasthan, India.

\section{Methods}

The dental practitioners were asked to complete a questionnaire regarding demographic data, prescribing practices, and knowledge about antibiotic use. A random sample of 525 dentists participated in the survey. The results were analysed using statistical software.

\section{Results}

Of the 525 surveys mailed,500 were returned.The respondents were registered dentists with mean age of $41 \mathrm{yrs}$. All of them were aware of the rising alarm on antibiotic misuse . They considered that the main causes of antibiotic resistance were patients not finishing the prescribed doses $(87 \%)$, wrong prescription $(80 \%)$, substandard doses $(67 \%)$, inefficient tools for diagnosing antibiotic resistance $(28 \%)$, lack of Govt. commitment to address these issues $(9 \%) \&$ poor surveillance $(8 \%)$. 
Respondents wrote an average of 5-10 prescriptions per day. The antibiotics they preferred to prescribe for use after treatment were : Amoxicillin - clavalunic acid (35\%); amoxicillin (33\%); ofloxacinornidazole $\quad(29 \%)$; ofloxacin ( $2 \%)$; clindamycin $(2 \%)$. The respondants prescribed antibiotics for a duration of three to seven days depending on the severity. The majority of respondents $(60 \%)$ prescribed antibiotics for 5 days, while $28 \%$ prescribed antibiotics for seven days. "Fig. 1 "

The percentage of dentists who prescribed antibiotics during various procedures were : $60 \%$ during RCT ; $18 \%$ before scaling ; $8 \%$ after scaling ; 40\% after root planing; $94 \%$ after abscess drainage ; $46 \%$ in case of gingival enlargement ; $86 \%$ after periodontal surgery; $76 \%$ single tooth extraction; $96 \%$ impacted tooth; $94 \%$ infection with fever \& $86 \%$ implant placement . "Fig 2"

The percentage of dentists who preferred to prescribe prophylactic antibiotic were : $90 \%$ in cardiovascular diseases, $78 \%$ in diabetics , $32 \%$ in pregnancy , $46 \%$ other systemic diseases \& $84 \%$ in infectious diseases. "Fig 3"

\section{Discussion}

Antibiotics are regularly prescribed by dentists for the management of oral and dental infections. Unfortunately their value is being jeopardized by the presence of microorganisms resistant to their effects [6].

In the present study, respondants reported following to be the main causes of antibiotic resistance : patients not finishing the prescribed doses (87\%), wrong prescription (80\%) , substandard doses $(67 \%)$, inefficient tools for diagnosing antibiotic resistance $(28 \%)$, lack of Govt. commitment to address these issues $(9 \%) \&$ poor survelliance $(8 \%)$.

Patients should be educated on the rising antibiotic resistance in the society, its importance \& the role they play in controlling it. The patient should be taught to complete the recommended prescribed doses. A regular surveillance should be conducted to monitor the susceptibility of microorganisms and detect any changes in their susceptibility. This information should be used to formulate strategies to combat antimicrobial resistance. By this we may also assess the impact of any strategies on resistance selection and transmission. Resistance trends, including the most prevalent mechanisms of resistance and their transmission, may also be determined from surveillance data. Govt . should also be committed to address these issues by making policy changes, formulary changes and alteration of prescribing practices [7].

Penicillin is still the gold standard in treating dental infections [8]. It is the most commonly prescribed antibiotics by dentists worldwide. However, there is less consensus regarding which drug belonging to this family should be prescribed. While some authors consider the natural and semisynthetic penicillins (amoxicillin) to be the options of first choice $[9,10]$, some penicillin V [11], others prefer the association amoxicillinclavulanate [12], due to the growing number of bacterial resistance, as well as its broad spectrum, pharmacokinetic profile, tolerance and dosing characteristics. Kirkwood [17] have proposed clindamycin as the drug of choice, in view of its good absorption, low incidence of bacterial resistances, and the high antibiotic concentrations reached in bone .

In the present survey, the most preferred antibiotic prescribed by dentists was amoxicillin - clavalunic acid combination for orodental infections . Rafael Roda et al has also shown it to be the most preferred drug by dentists during 2005, at least in the Valencian Community (Spain) [12] .

Liñares and Martin-Herrero [13] had considered amoxicillin-clavulanate to be the option with the fewest resistant strains. Sobottka et al. [14] isolated 87 pathogens from 37 patients with odontogenic abscesses, found $100 \%$ to be sensitive to amoxicillin-clavulanic acid. Kuriyama et al 2000 [15] found no difference in clinical outcome between penicillin $\mathrm{V}$, amoxicillin, or amoxicillin and clavulanate.

Amoxicillin was the second most common antibiotic preferred in the present study. It was also the most preferred antibiotic by Roy \& Bragg [16], Palmer et al 2000 [9]. Liñares and Martin-Herrero have shown that amoxicillin shows resistance in 30-80\% of all strains of Prevotella and Porphyromona [13].

Ofloxacin - Ornidazole combination ofloxacin \& metronidazole were the other antimicrobials prescribed . Sobottka et al [14] showed excellent results with fluorquinolones (moxifloxacin and levofloxacin), with sensitivity in $98 \%$ of all strains in treating odontogenic abscesses. Kirkwood [17] considered that although the penicillins traditionally have been used for the treatment of odontogenic infections, the growing presence of bacteria resistant to penicillin have caused other antibacterials to become the drugs of choice i.e.clindamycin. In contrast to the above, Swift et al [18] indicate that despite the recent introduction of many new antimicrobials, none have demonstrated significant benefit justifying their replacement of penicillin derivatives in application to orofacial infections. Furthermore, they consider that the appropriate use of these drugs, together with surgery, constitute adequate treatment for odontogenic infections.

Antibiotics should be prescribed at the correct frequency, dose, and duration so that the minimal inhibitory concentration is exceeded, and so that side effects and the selection of resistant bacteria are prevented[18]. The majority of respondents $(60 \%)$ in the present study prescribed antibiotics for 5 days, while $28 \%$ prescribed antibiotics for seven days. Roy \& Bragg reported that more than half of all the prescriptions 
written in Glasggow, Scotland were for a course of 5 days or more. A survey in Canada found that the average duration of antibiotic use prescribed by dentists is 6.92 days [19] . Another survey found in the USA found that endodontists prescribe antibiotic use for an average of 7.58 days [20]. Contrary to this current views have shown the merits of shorter courses [21]. Prolonged courses of antibiotics destroy the commensal flora [22]. In addition, longer durations of up to 21 days may result in the selection of resistant strains and a reduction in the ability of the oral flora to resist the colonization by harmful micro-organisms that are not normal residents, [23] leading to superimposed infections by multi-resistant bacteria and yeasts.

Antibiotics are typically prescribed in dental practice for some of the following purposes: (a) as treatment for acute odontogenic infections; (b) as treatment for non-odontogenic infections; (c) as prophylaxis against focal infection in patients at risk (endocarditis and joint prostheses); and (d) as prophylaxis against local infection and systemic spread in oral surgery. Rational antibiotic use is thus required in dental and oral clinical practice, to ensure maximum efficacy while at the same time minimizing the side effects and the appearance of resistances [12].

The percentage of dentists who prescribed antibiotics during various procedures were : $60 \%$ during RCT ; 18\% before scaling ; $8 \%$ after scaling ; $40 \%$ after root planing; $94 \%$ after abscess drainage ; $46 \%$ gingival enlargement ; $86 \%$ after periodontal surgery; $76 \%$ single tooth extraction; $96 \%$ impacted tooth; $94 \%$ infection with fever \& $86 \%$ implant placement .

Therapeutic prescribing of antibiotics in the present study showed that antibiotics were also prescribed in non indicated clinical cases like before \& after scaling \& root planing, during endodontic treatments, gingival enlargements. The most common orodental infections are pulpitis and periapical periodontitis, which require only operative measures like fillings, root canal therapy, or extraction if the tooth is not restorable. American dental association in 1997 stated that the routine use of antibiotics before or after extractions or endodontics is not effective. Therefore, routine prescribing for every extraction or endodontic procedure must be discouraged . Unfortunately, dentists still prescribe antibiotics for this condition [9,11,20,24]. Clinically indicated cases for antibiotic use are periodontal \& oral infections accompanied by fever \& evidence of systemic spread [8], periodontal abscesses, acute necrotising ulcerative gingivitis \& pericoronitis [24]. Before prescribing antibiotics, clinician should weigh the risks of resistance and allergy against any potential benefit. Some adverse reactions include allergic / anaphylactic reactions, superinfections of oppurtunistic bacteria, interactions with other medications, upset stomach, nausea \& vomiting. Our decisions on antibiotic use should be supported by the current literature, which is continually being updated as bacteria develop new "tricks" to cope with antibiotics.

Prophylactic use of antibiotics has been advocated prior to a number of dental procedures, to reduce the likelihood of postoperative local complications, like infection, dry socket, or serious systemic complications like infective endocarditis. In the case of bacterial endocarditis (IE), the absolute risk rate after dental treatment, even in at-risk patients, is considered very low [25]. This is consistent with recent guidelines from the British Society for Antimicrobial Chemotherapy [26], and the American Heart Association [27], which recommend that only patients in the high risk category require cover. The basis for this recommendation is:

- There is no consistent association between having an intervention, dental or non-dental, and the development of IE.

- Regular tooth brushing almost certainly presents a greater risk of IE than a single dental procedure because of repetitive exposure to bacteremia with oral flora.

- The clinical effectiveness of antibiotic prophylaxis is not proven.

- Antibiotic prophylaxis against IE for dental procedures may lead to a greater number of deaths through fatal anaphylaxis than would a strategy of no antibiotic prophylaxis. Nor is antibiotic prophylaxis against IE cost effective.

The American Heart Association recommends prophylaxis against infective endocarditis in cardiac patients only in dental procedures associated with significant bleeding. Such procedures include dental extractions, periodontal surgery and scaling. Reassuringly, the majority of dentists surveyed by Palmer et al.[9] would all prescribe prophylaxis for patients with cardiac conditions undergoing these procedures. High-risk patients include those with prosthetic heart valves, previous bacterial endocarditis or complex cyanotic congenital heart disease. Patients with a past history of rheumatic fever without valvular dysfunction are at negligible risk of infective endocarditis.

Of all the dentists surveyed in the present study $90 \%$ reported giving prophylaxis in the various cardiovascular conditions , $46 \%$ in other systemic diseases \& $84 \%$ in infectious diseases. . Antibiotics were also given in non indicated conditions like pregnancy $(36 \%)$.

Therefore, antibiotic use must be rational \& moderate to reduce the development of cross resistance \& needless patient exposure . Currently, an ideal antibiotic for the treatment of dental diseases does not exist . Although oral bacteria are susceptible to many antibiotics, no single antibiotic at concentrations achieved in body fluids inhibits all putative pathogens. Indeed , a combination of antibiotics may be necessary to eliminate 
all putative pathogens. When used to treat orodental infections, antibiotic should be selected on the basis of patients medical \& dental status, current medications \& results of microbial analysis .

The diagnostic microbiology laboratory also has a significant role to play in surveillance, through identification of organisms with a pathogenic role in infection, and their subsequent susceptibility testing. It is therefore important to establish better awareness and communication between general dental practitioners .

Figures and Tables(11 Bold)

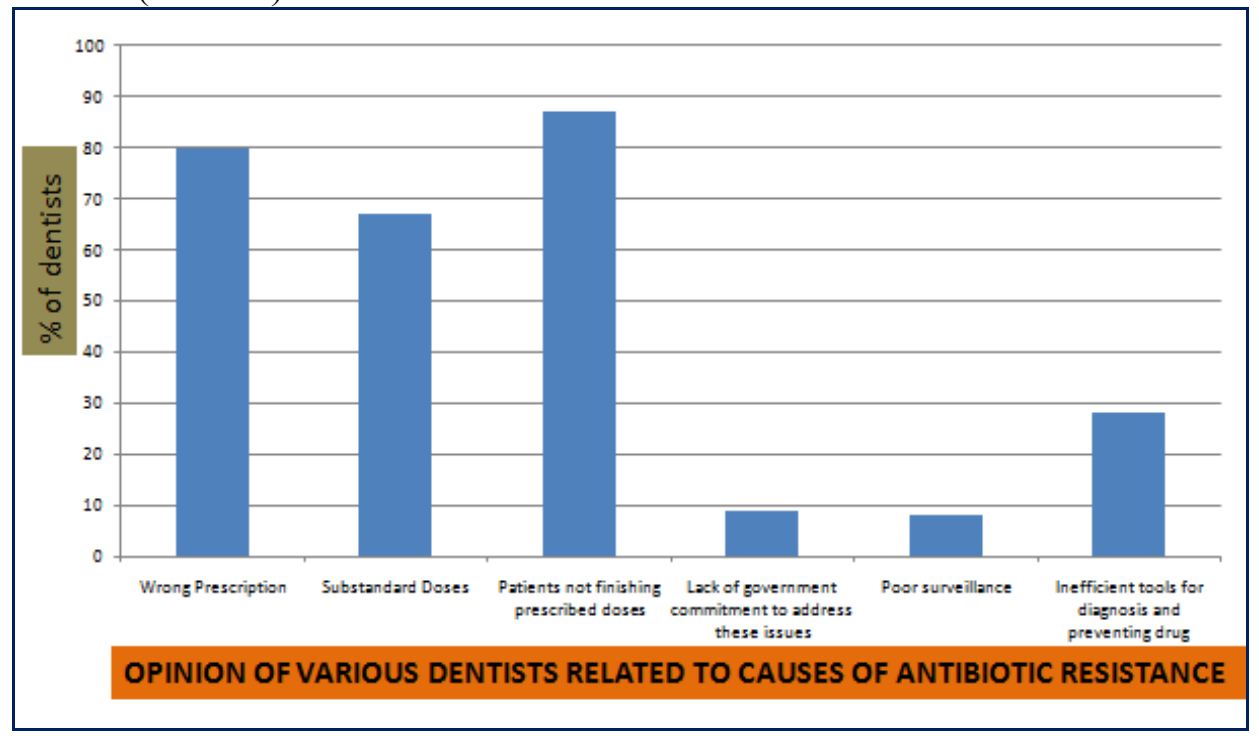

Fig 1 opinion of various dentists related to causes of antibiotic resistance

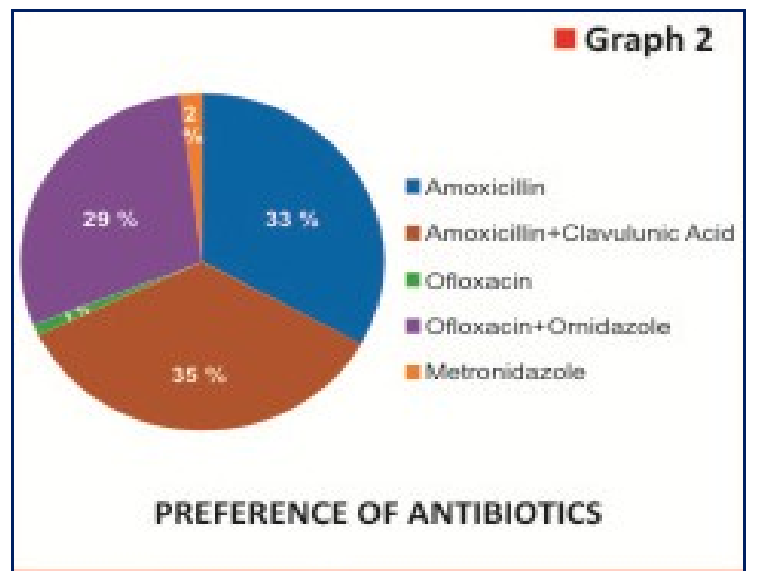

Fig. 2 preference of antibiotics

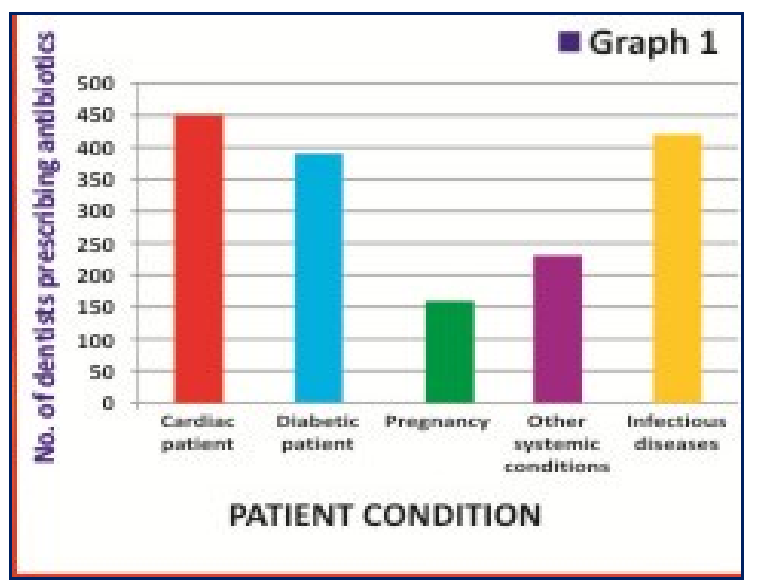

Fig.3 no.of dentists prescribing antibiotics in various patient conditions 


\section{Conclusion}

There is evidence that inappropriate prescribing of antibiotics by dental practitioners may be contributing to the development of the antibiotic resistance. If so, then the dental profession must take steps to prevent the problem from becoming worse. Comprehensive undergraduate and postgraduate education on the subject is required. Further studies and establishment of a surveillance system are also to be recommended. Clear guidelines and prescribing policies need to be developed to attempt to limit resistance within the oral flora. Antibiotic use by dentists affects flora that exist beyond the oral cavity, since, during therapy, antibiotics become distributed throughout the body. Antibiotics prescribed by medical practitioners also have similar broad effects. If dentists, along with medical practitioners, can reduce the number of antibiotic prescriptions, the rate of resistance development may be slowed.

The installation of a surveillance system, accompanied by audit to ascertain the number of prescriptions written and their appropriateness, to highlight areas of prescribing knowledge that are lacking in dental practice are recommended. Although it must be remembered that prescriptions are an indirect measure of antibiotic consumption and are not a measure of the rate of resistance emergence, which is multifactorial. Further investigation and education are required to attempt to slow resistance development and lessen the future impact on antibiotic prescribing in dentistry. The emergence of antibiotic-resistant bacteria within the oral flora will have an impact on the prescribing of antibiotics in dentistry.

\section{References}

[1] Greenstein G, Changing periodontal concepts : treatment considerations , Compend Contin Educ Dent, 26( 81) ,2005

[2] Najla Saeed Dar-Odeh,Osama Abdalla Abu-Hammad,Mahmoud Khaled Al-Omiri,Ameen Sameh Khraisat and Asem Ata Shehabi, Antibiotic prescribing practices by dentists: a review, Ther Clin Risk Manag, 2010, (6) , 301-306.

[3] Lewis MA, Why we must reduce dental prescription of antibiotics, European Union Antibiotic Awareness Day, Br Dent J. 2008, 205(10), 537-8.

[4] Ann R, The use of systemic antibiotics in dental practice , Australas Coll Dent Surg 1998,14, 62-5.

[5] World Health Organization symposium. Monitoring and management of bacterial resistance to antimicrobial agents: A world Health Organization Symposium. Clin Infec Dis 24(Suppl 1)1997, S 1-176.

[6] Cohen FL \& Tartasky U, Microbial resistance to drug therapy: A review, Am J Infect Control 1997,25, 51-64.

[7] ADA Council on scientific affairs combating antibiotic resistance. Association report, J Am Dent Assoc 2000, $135(4), 484-487$.

[8] Swift JQ, Gulden WS, Antibiotic therapy - managing odontogenic infections, Dent Clin N Am. 2002,46,623-633.

[9] Palmer NO, Martin MV, Pealing R, Ireland RS, An analysis of antibiotic prescriptions from general dental practitioners in England , J Antimicrob Chemother. 2000, 46, 1033-1035.

[10] Sarkar C, Das B, Baral P, An audit of drug prescribing practices of dentists, Indian J Dent Res. 2004,15(2),58-61.

[11] Demirbas F, Gjermo PE, Preus HR , Antibiotic prescribing practices among Norwegian dentists , Acta Odontol Scand. 2006 , 64(6), 355-359

[12] Rafael Poveda Roda, José Vicente Bagán, José María Sanchis Bielsa ,Enrique Carbonell Pastor, Antibiotic use in dental practice. A review, Med Oral Patol Oral Cir Bucal 2007; 12, E186-92.

[13] Liñares R, Martín-Herrero JE, Bases farmacológicas del tratamiento antibiótico de las enfermedades periodontales y periimplantarias, Av Odontoestomatol 2003, especial , 23-33.

[14] Sobottka I, Cachovan G, Sturenburg E, Ahlers MO, Laufs R, Platzer U, In vitro activity of moxifloxacin against bacteria isolated from odontogenic abscesses, Antimicrob Agents Chemother 2002 , 46,4019-21.

[15] Kuriyama T, Nakagawa K, Karasawa T, Saiki Y, Yamamoto E, Nakamura S. Past administration of beta-lactam antibiotics and increase in the emergence of beta-lactamase-producing bacteria in patients with orofacial odontogenic infections. Oral Surg Oral Med Oral Pathol Oral Radiol Endod 2000,89,186-92.

[16] K. M. Roy and J. Bagg, Antibiotic prescribing by general dental practitioners in the Greater Glasgow Health Board, Scotland, Br Dent J 2000, 188(12),674-676.

[17] Kirkwood KL, Update on antibiotics used to treat orofacial infections, Alpha Omegan 2003, 96, 28-34.

[18] Guillemot D, Carbon C, vauzellekervroedan F , Inappropriateness and variability of antibiotic prescription among French officebased physicians, J Clin Epidemiol 1998, 51, 61-68.

[19] Epstein JB, Chong S, Le ND , A survey of antibiotic use in dentistry , J Am Dent Assoc. 2000, 131(11), 1600-1609.

[20] Yingling NM, Byrne BE, Hartwell GR , Antibiotic use by members of the American Association of Endodontists in the year 2000: report of a national survey, J Endod. 2002, 28(5), 396-404.

[21] Slots J, Systematic antibiotics in periodontics, J Perio 1996, 67, 831-838.

[22] Bax R, Development of a twice daily dosing regimen of amoxicillin/clavulanate, Int J Antimicrob Agents. 2007, 30(Suppl 2), S118-S121.

[23] Longman LP, Martin MV. The use of antibiotics in the prevention of postoperative infection: a re-appraisal. Br Dent J. 1991;170:257-262

[24] Salako N, Rotimi VO, Adib SM, Al-Mutawa S , Pattern of antibiotic prescription in the management of oral diseases among dentists in Kuwait, J Dent. 2004, 32, 503-509.

[25] Pallasch TJ, Antibiotic resistance, Dent Clin N Am. 2003, 47,623-639

[26] Gould FK, Elliott TS, Foweraker J , Guidelines for the prevention of endocarditis: report of the Working Party of the British Society for Antimicrobial Chemotherapy, J Antimicrob Chemother. 2006,57(6),1035-1042.

[27] Wilson W, Taubert KA, Gewitz M, Prevention of infective endocarditis: guidelines from the American Heart Association: a guideline from the American Heart Association Rheumatic Fever, Endocarditis, and Kawasaki Disease Committee, Council on Cardiovascular Disease in the Young, and the Council on Clinical Cardiology, Council on Cardiovascular Surgery and Anesthesia, and the Quality of Care and Outcomes Research Interdisciplinary Working Group, Circulation. 2007,116(15),1736-1754. 